\title{
Producción de enzimas extracelulares por bacterias aisladas de invertebrados marinos
}

\author{
Extracellular enzymes produced by bacteria isolated from marine invertebrates
}

Jorge León, Fabiola Pellón, Verónica Unda, Janeth David, César Anaya y Victoria Mendoza*

\begin{abstract}
in order to select marine bacteria with the ability to produce extracellular enzymes (EEC), samples from Argopecten purpuratus and Crassostrea gigas in cultivation as well as from other intertidal and benthic invertebrates were analyzed. The selection of producer EEC strains was carried out in Marine Agar (MA) with addition of the relevant substratum (starch, casein, tween-80, lecithine, DNA and gelatine). The EEC producer strains evaluation was carried out on 102 isolates.

The results show that bacteria associated to $A$. purpuratus and $C$. gigas have the best multienzymatic activities; however, Semimytilus algosus, Tetrapigus niger and Thais chocolata are also important sources of bacteria producers of EEC. The qualitative multienzymatic activity in MA (clear or opaque zones around the colonies in $\mathrm{mm}$ of diameter) varied from 6 to $>16 \mathrm{~mm}$. The frequency of multienzymatic production was caseinase $62,74 \%$, tween-esterase $57,84 \%$, amylase $52,94 \%$, gelatinase $38,23 \%$, DNAse $33,3 \%$, agarase $5,43 \%$ and lecithinase $90,0 \%$. The results suggest the possibility of using native strains for biotechnological purposes
\end{abstract}

Key words: extracellular enzymes, metabolites, marine bacteria, invertebrate, marine biotechnology

En ambientes marinos, la búsqueda y aislamiento de cepas de bacterias nativas productoras de substancias bioactivas se ha realizado a partir de diversas muestras (Martin, 1976; Martin y Bianchi, 1980; Prieur, 1989). Entie estas substancias destacan las "Enzimas Extracelulares" (EEC), cuyo desarrollo en el sector industrial se ha producido en forma explosiva en los últimos años (Chandrasekaran, 1997). Las enzimas nicrobianas procedentes de microorganismos de ambientes acuáticos incluyen amilasas, glucamilasas, glucosaisomerasas, proteasas, pectinasas (Stanley \& Stanley, 1986) y otros como agarasas, quitinasas, alginasas, lipasas, Dnawals y esterasas (Fenical y Jensen. 1993). La mayoría de estas enzimas tienen aplica-

Microbiologia Ambiental y Bwolecnologia, Faculad de Ciencias Biologicas Universidad Nacional Mayor do San Marcos, Lima. Perú.

E-mail:0190026 bunmsm edupe ción biotecnológica, especialmente en la industria alimentaria (Stanley y Sianley, 1986).

Las enzimas proteolíticas y lipolíticas actúan sobre diversos subsilalus naturales y sintéticos y son principalmente utilizadas en la industria como detergentus, en tanto las amilasas, gelatinasas, caseinasas y agarasas se empican como aditivos en la industria alimentaria (Barzana y López-Munguía, 1995).

El presente estudio tiene como objetivo central seleccionar capas nativas de bacterias marinas hiperproductoras de EEC. Pura ello, muestras de invertebrados intermareales y bentónicos fueron recolectadas en la Rahía de Ancón "San Francisco" y "Punta LA CRUZ", respectivamente). Asimismo, muestras de Argopecien purpuratus "concha de abanico" y Crassostrea gigas "ostra" fue- 
ron recolectadas en las zonas de cultivo "El Carbón" y "La Tiza" (Bahía de Pucusana) y en la Isla San Lorenzo (Callao). Los organismos intermareales y bentónicos fueron obtenidos mediante recolección directa y por buceo respectivamente, y las muestras de Argopecien purpuralus y Crassostrea gigas fueron tomadas directamente de las "linternas de cultivo". Las muestras fueron procesadas según la metodología descrita por León (1996), utilizando el Agar Marino de ZoBell (AM) como medio de aislamiento bacteriano.

Para la selección de cepas productoras de EEC, se utilizó el método de formación de "macrocolonias". El AM sirvió como medio base al cual se incorporaron los substratos correspondientes (almidón, caseína, tween- 80 . lecitina y DNA) en una concentración de $1 \%$ $(\mathrm{p} / \mathrm{p})$. La actividad frente a la gelatina fue evaluada en el medio AM con adición de gelatina microbiológica al $12 \%(\mathrm{p} / \mathrm{p})$. Las cepas cultivadas fueron incubadas a $22^{\circ} \mathrm{C}$ hasta por 10 días. La actividad enzimática de las cepas sobre los substratos en pruebá fue determinada por la presencia y tamaño de las zonas de hidrólisis alrededor de las macrocolonias. Asimismo, 6 cepas aisladas de Argopecten purpuratus y 4 de Crassostrea gigas en cultivos fuelon identificadas a nivel de género utilizando esquemas de identificación para bacterias marinas según Oliver (1982) y Sawabe et al. (1995).

Se aislaron un total de 102 cepas de bacterias $(45$ provienen de organismos intermareales, 47 de bentónicos, 6 de Argopecten purpuratus y 4 de Cicrssostrea gigas), las cuales fueron evaluadas por su capacidad de producir substancias con actividad multienzimática (Tabla 1, 2 y 3). La acción enzimática de las cepas en estudio sobre diversos substratos muestra resultados homogéneos. Sin embargo, cabe resaltar que las cepas provenientes de Semimyilus algosus "chorito negro" (cepa Chol-Al) y de Tetrapigus niger "erizo negro" (cepas Eri5AI y Eri7-AI) cuentan con mejor actividad multienzimática, llegando inclusive a actuar frente a 5 substratos diferentes. siendo las más importantes la actividad amilolítica (amilasa). proteolítica (caseinasa) y lipolítica (tween esterasa) (Tabla I). Las cepas provenientes de Argopecteri purpuratus de vida libre (cepa Cab3-AII), Tetrapigus niger "erizo negro" (cepas Eri2-AII, Eri3-AII) y Thais chocolata "caracol" (cepa Cchl-AII) mostraron igualmente mayor actividad multienzimática (Tabla 2). Resultados similares fueron obtenidos por Harris (1993), quien analizó la actividad multienzimática de bacterias aisladas del tracto intestinal de varias especies de invertebrados acuáticos, demostrando que una gran proporción de crustáceos y moluscos estudiados eran portadores de bacterias con actividades enzimáticas principalmente proteolíticas, lipolíticas y quitinolíticas; en cambio, las bacterias celulolíticas eran escasas. Relacionado a este último, queda por investigar la relación de ciertos miembros de la microflora intestinal capaces de producir exoenzimas con los requerimientos alimenticios de especies de invertebrados hervíboros y detritíboros. En el presente estudio también se reporta la actividad agarolítica de ciertas cepas; sin embargo, fueron pocas las bacterias aisladas con capacidad de producir agarasas. Al respecto, León et al. (1998) en aislamientos previos lograron obtener cepas hiperproductoras de agarasas a partir de otros invertebrados intermareales.

La Tabla 3 y Figuras 1, 2, 3, 4, 5 y 6 muestran los resultados de la actividad multienzimática de EEC de 10 cepas provenientes de Argopecten purpuratus y Crassostrea gigasen cultivos (Pellón, 2000). Cabe resaltar que la tolalidad de las cepas mostraron actividades más contundentes com- 
paradas con aquellas producidas por cepas provenientes de organismos intermareales y bentónicos. La identificación preliminar de estas cepas determinó a Vibrio como el género que predomina. Otras cepas identificadas pertenecen a los géneros Flavobacterium, Moraxella y Flexibacter.

Es importante resaltar que de un total de 102 aislados (100\%) previamente seleccionados por tener alguna actividad enzimática extracelular, la frecuencia de cepas que presentan actividades multienzimáticas es como sigue: caseinasa 62,74 , tween-esterasa $57,84 \%$, amilasa $52,94 \%$, gelatinasa $38,23 \%$ y DNAsa $33,3 \%$. La producción de agarasa $(5,43 \%)$ y lecitinasa (90\%) fue evaluada en 92 y 10 cepas respectivamente (Tabla 4). Al respecto, Marty y Martín (1992), estudiando bacterias marinas asociadas a organismos marinos (cepas epibiontas), aislaron numerosas cepas nativas productoras de exoenzimas. Por su parte, León (1996) aisló cepas de Pseudomonas, Vibrio, Alteromonas, Flavobacterium, Cytophaga y Micrococcus provenientes del neuston marino que, además de mostrar actividades inhibitorias de amplio espectro frente a bacterias ictiopatógenas, también evidenciaron capacidad de producir EEC. Fn este estudio, nuestros resultados muestran la presencia de numerosas cepas nativas productoras de EEC con actividad multienzimática asociadas a invertebrados poco explorados. Estas propiedades de las bacterias marinas podrían ser aprovechadas para un posible o eventual explotación biotecnológica. Estudios subsiguientes determinarán las potencialidades microbianas de origen marino de producción de otras enzimas de intcrés industrial (quitinasas, celulasas, pronasas, alginasas y otras).

\section{AGRADECIMIENTIOS}

Al Consejo Superior de Investigaciones de la UNMSM, por su financiamiento econónico a través del Proyecto 91001171-1999. Asi- mismo, al equipo de Investigaciones Marinas Grupo DePSEA (Laboratorio de Ecología Marina, Facultad de Ciencias Biológicas, UNMSM) a cargo del Dr. Juan Tarazona B. por su valiosa colaboración en la obtención de muestras bentónicas.

\section{LITERATURA CITADA}

Bárzana, E. y A. López-Munguía. 1995. La tecnología enzimática. En Biotecnología Alimentaria, pp. 103-123. Limusa, México D.i.

Chandrasekaran, M. 1997. Industrial enzymes from marine microorganisms: The Indian scenario. J. Mar. Biotechnol. 5:86-89.

Fenical, W. y PR. Jensen. 1993. Marine microorganisms: a new biomedical resource. In Marine Biotechnology. Pharmaceutical and Bioactive Natural Products. Edited by Attaway DH, Zaborsky OR, New York: Plenum Press, I: 419-457.

Harris, JM. 1993. The presence, nature, and role of gut microflora in aquatic invertebrates: a synthesis. Microb. Ecol. 25: 195-231.

León, J. 1996. Cepas nativas del bacterioneuston marino con actividad antagónica frente a bacterias ictiopatógenas. Caractcrización preliminar de substancias inhibitorias. Tesis Mg. Sc. Universidad Católica de Valparaíso, Chile.

León, J, P. Ramírez, M. Alcarráz y E. Castro. 1998. Bacterias marinas asociadas a invertebrados: Evaluación del efecto inhibitorio frente a patógenos de peces, noluscos y crustáceos. Libro de Resumen de la VII Reunión 
Científica de JCBAR, UNMSM.pp. 77.

Martin, Y. P. 1976. Importance des bactéries chez les mollusques bivalves-Haliotis, 1976-78, volume 7: 97-103

Martin, Y. P y M. A. Bianchi. 1980. Structure, diversity and catabolic potentialities of aerobic heterotrophic bacterial populations associated with continuous cultures of natural marine phytoplankton. Microb. Ecology., 5: 265-279.

Marty, P. y Y. Martin. 1992. Aerobic heterotrophic bacteria associated with some Mediterranean coastal benthic invertebrates: Characterization of strains, exoenzyme and antibiotic production. Mar Life. I(1): 1.8.

Oliver, J. D. 1982. Taxonomic scheme for the identification of marine bacteria. Deep-Sea Res. 29: 795-98.

Pellón, F. 2000. Aislamiento y caracterización de bacterias marinas con capacidad antibacteriana, asociadas a moluscos bivalvos en cultivos. Tesis para optar al Título Profesional de Biólogo con mención en MicrobiologíaParasitología, Facultad de Ciencias Biológicas, LNMSM.

Prieur. D. 1989. Deep-sea hydrothermal vents of $13^{\circ} \mathrm{N}$ (East Pacific Rise): preliminary bacterial survey and biotechnological potential. In: Microbiology of extreme environments and its potential for biotechnology. Da Costa, Duarte and Williams Eds.. Elsevier Applied Sciences, London and New York: 163-166.

Sawabe, T, Y. Oda, Y. Shiomi y Y. Ezura. 1995. Alginate degradation by bacteria isolated from the gut of sea urchins and abalones. Microb. Ecol. 30: 193 202.

Stanley, I. T y P. M. Stanley. 1986. Potential commercial applications in aquatic microbiology. Microb. Ecol., 12:79100. 
Tabla 1. Actividad multienzimática de 45 cepas de bacterias marinas aisladas de invertebrados intermareales recolectados en la Bahia de Ancón, Lima, Perú, 1999

\begin{tabular}{|c|c|c|c|c|c|c|c|}
\hline \multirow{2}{*}{ Invertebrados } & \multirow{2}{*}{$\begin{array}{c}\text { Cepas aisladas } \\
(n=45)\end{array}$} & \multicolumn{6}{|c|}{ A tividad enzimática (") } \\
\hline & & 1 & 2 & 3 & 4 & 5 & 6 \\
\hline \multirow{4}{*}{$\begin{array}{l}\text { Temistes hemaghe } \\
\text { "pepino de mar" }\end{array}$} & P1-Al & - & + & - & - & - & + \\
\hline & $\mathrm{P} 2-\mathrm{Al}$ & - & - & - & $\cdot$ & - & + \\
\hline & P3-Al & ++ & - & ++ & - & - & + \\
\hline & P4-Al & - & - & + & - & - & - \\
\hline \multirow{2}{*}{$\begin{array}{l}\text { Chiton cumingsii } \\
\text { "chiton" }\end{array}$} & $\operatorname{Ch}|-A|$ & - & - & - & - & + & - \\
\hline & Ch2-Al & $+t$ & - & ++ & ++ & - & $\cdot$ \\
\hline \multirow{3}{*}{$\begin{array}{l}\text { Semimytilus algosus } \\
\text { "chorito negro" }\end{array}$} & Cho1-Al & + & $++t$ & ++ & ++ & $+t+$ & - \\
\hline & Cho2-Al & + & +++ & - & $\cdot$ & - & - \\
\hline & Cho4-Al & + & + & - & - & - & - \\
\hline \multirow{3}{*}{$\begin{array}{l}\text { Emerila analoga } \\
\text { "muymuy" }\end{array}$} & $M m i-A l$ & + & ++ & +++ & - & +++ & - \\
\hline & $\mathrm{Mm} 2 \cdot \mathrm{Al}$ & + & - & + & - & · & - \\
\hline & Mm3-Al & $\cdot$ & $\cdot$ & +++ & - & - & - \\
\hline \multirow{4}{*}{$\begin{array}{l}\text { Geograpsus lividus } \\
\text { "cangrejo" }\end{array}$} & $\operatorname{Cg}|A|$ & - & - & + & . & - & - \\
\hline & $\operatorname{Cg} 2 \cdot A 1$ & ++ & ++ & - & - & - & - \\
\hline & Cg 3-Al & - & - & $+t$ & ++ & $\cdot$ & $\cdot$ \\
\hline & $\operatorname{Cg} 4-\mathrm{Al}$ & - & +++ & ++ & - & ++ & $\cdot$ \\
\hline \multirow{4}{*}{$\begin{array}{l}\text { Tegula atra } \\
\text { "caracol turbante" }\end{array}$} & $\operatorname{cg} 5 \cdot A 1$ & $+t$ & +++ & + & + & ++ & $\cdot$ \\
\hline & $\operatorname{Con} 1-A \mid$ & + & $+t$ & ++ & - & - & - \\
\hline & Cch2-Al & +++ & - & + & - & + & - \\
\hline & $\operatorname{Cch} 3-A l$ & $\cdot$ & + & $\cdot$ & + & - & - \\
\hline \multirow{3}{*}{ Ophiactys sp } & Es 1-Al & - & - & + & - & - & - \\
\hline & Es2-Al & . & - & + & - & - & $\cdot$ \\
\hline & Es3-Al & + & + & ++ & + & - & - \\
\hline \multirow{3}{*}{ Balanus laevis } & $\mathrm{Ci2}-\mathrm{Al}$ & - & ++ & - & - & . & . \\
\hline & $\mathrm{O} 3-\mathrm{Al}$ & $+t$ & $+t$ & - & ++ & + & - \\
\hline & $\mathrm{Ci} 4-\mathrm{Al}$ & - & - & $+t$ & - & + & - \\
\hline \multirow{8}{*}{$\begin{array}{l}\text { Tetrapigus niger } \\
\text { "enzo de mar" }\end{array}$} & Eri1-Al & +++ & $+t$ & ++ & - & - & - \\
\hline & Eri2-Al & + & ++ & ++ & - & + & - \\
\hline & Eri3-Al & - & + & - & . & - & - \\
\hline & Eri4-Al & + & + & + & - & $\cdot$ & - \\
\hline & Eri5-Al & +++ & ++ & $+t$ & ++ & ++ & - \\
\hline & Eric-Al & - & ++ & ++ & - & - & - \\
\hline & Eri7-Al & + & +++ & +++ & + & ++ & - \\
\hline & Eris-Al & + & ++ & ++ & - & ++ & - \\
\hline \multirow{11}{*}{$\begin{array}{l}\text { Phymactis clematis } \\
\text { "anémona de mar" }\end{array}$} & Anm1-Al & - & +++ & t+ & ++ & - & - \\
\hline & $A n m 2-A \mid$ & - & - & + & - & . & - \\
\hline & Anm3-Al & +++ & - & + & - & - & - \\
\hline & AnmA-Al & +++ & - & ++ & - & - & - \\
\hline & Anm5-Al & - & + & - & - & - & - \\
\hline & $A \cap m 6-A l$ & - & - & ++ & - & - & - \\
\hline & Anm $7-A !$ & ++ & + & + & - & $+t$ & - \\
\hline & Anm8-A & $\cdot$ & + & $\cdot$ & $\cdot$ & - & - \\
\hline & $A \cap m 9-A \mid$ & - & t+ & - & ++ & - & $\cdot$ \\
\hline & Anm10-Al & + & $\cdot$ & - & - & + & - \\
\hline & Anm11-Al & - & - & ++ & $\cdot$ & - & - \\
\hline
\end{tabular}


Tabla 2. Activiơad multienzimática de bacterias marinas aisladas de invertebrados bentónicos de "Punta Cruz", Ancón, Lima, Perú. 1999

\begin{tabular}{|c|c|c|c|c|c|c|c|}
\hline \multirow{2}{*}{ Invertebrados } & \multirow{2}{*}{$\begin{array}{c}\text { Cepas aisladas } \\
47\end{array}$} & \multicolumn{6}{|c|}{ A tividad enzimática (*) } \\
\hline & & 1 & 2 & 3 & 4 & 5 & 6 \\
\hline \multirow{3}{*}{$\begin{array}{l}\text { Argopecten purpuralus } \\
\text { "concha de abanico" }\end{array}$} & Cab1-All & $\cdot$ & $\cdot$ & + & $\cdot$ & - & - \\
\hline & Cab2-All & - & - & - & ++ & - & - \\
\hline & Cab3-All & +++ & +++ & ++ & + & - & - \\
\hline \multirow{10}{*}{$\begin{array}{l}\text { Thais cho colata } \\
\text { "caracol" }\end{array}$} & $\operatorname{cch} 1 \cdot$ All & +++ & ++ & . & + & $++t$ & - \\
\hline & $\operatorname{Cch} 2 \cdot A l l$ & ++ & + & . & - & - & - \\
\hline & Cch3-All & - & + & - & $\cdot$ & - & - \\
\hline & Cch4.All & - & - & . & + & - & - \\
\hline & $\operatorname{Cch} 5$ All & - & + & - & - & + & - \\
\hline & Cch6-All & - & - & - & ++ & - & - \\
\hline & Cch7-All & - & ++ & - & $\cdot$ & ++ & - \\
\hline & Cch8-All & + & $++t$ & - & t+ & - & - \\
\hline & Cch $9-A l l$ & & + & - & $\cdot$ & - & - \\
\hline & Cch10-All & . & ++ & - & - & - & . \\
\hline \multirow{5}{*}{$\begin{array}{l}\text { Te lrapigus niger } \\
\text { "erizo negro" }\end{array}$} & Eri1-All & - & ++ & - & - & ++ & + \\
\hline & Eri2-All & ++ & ++ & ++ & - & ++ & . \\
\hline & Eri3-All & ++ & $+t$ & ++ & $+t$ & $\cdot$ & . \\
\hline & Eris-All & - & ++ & - & ++ & - & + \\
\hline & Eri5-All & - & $\cdot$ & $\cdot$ & - & + & - \\
\hline \multirow{6}{*}{$\begin{array}{l}\text { Tegula atra } \\
\text { "caracol lurbante" }\end{array}$} & Cgni-All & $+t$ & - & ++ & . & - & . \\
\hline & $\operatorname{Cgn} 2-A l l$ & +++ & - & ++ & $\cdot$ & - & . \\
\hline & $\mathrm{Cg} \cap 3 \cdot \mathrm{All}$ & - & $+t$ & + & $\cdot$ & - & - \\
\hline & Cgnit-All & - & ++ & ++ & - & ++ & . \\
\hline & $\operatorname{Cg} n 5-A \|$ & - & ++ & ++ & . & ++ & . \\
\hline & $\operatorname{Cgn} 6-A \|$ & - & ++ & - & - & ++ & - \\
\hline \multirow{4}{*}{$\begin{array}{l}\text { Chondracanthus } \\
\text { chamisoi (a) }\end{array}$} & $A \mid g\lfloor-A \|$ & . & +++ & ++ & ++ & ++ & - \\
\hline & Alg2-All & + & $+t+$ & - & tt & - & - \\
\hline & Alg3-All & - & +++ & + & ++ & ++ & - \\
\hline & Alg4-All & $\cdot$ & $t+t$ & - & - & + & - \\
\hline \multirow{2}{*}{$\begin{array}{l}\text { Heliaster hefianthus } \\
\text { "estrella sol" }\end{array}$} & Es 1.All & + & - & - & - & . & . \\
\hline & Es2-All & - & - & ++ & . & - & . \\
\hline \multirow{5}{*}{$\begin{array}{l}\text { Thais hae mostoma } \\
\text { "caracol" }\end{array}$} & Cari-All & $\cdot$ & + & - & + & - & - \\
\hline & Car2-All & ++ & $\cdot$ & ++ & - & $\cdot$ & - \\
\hline & Car3-All & $\cdot$ & +++ & - & ++ & ++ & . \\
\hline & Car4-All & $t++$ & $\cdot$ & +++ & $\cdot$ & - & - \\
\hline & Car5-All & $\cdot$ & ++ & - & - & ++ & - \\
\hline \multirow{5}{*}{$\begin{array}{l}\text { Sinum cymba } \\
\text { "caracol" }\end{array}$} & $X 1-A l l$ & - & + & . & . & . & - \\
\hline & $X 2-A l l$ & ++ & - & - & - & - & - \\
\hline & X3-All & ++ & - & - & - & $\cdot$ & $\cdot$ \\
\hline & X4 -AH & ++ & $+t$ & - & - & + & \\
\hline & X5-All & + & - & $\cdot$ & - & $\cdot$ & - \\
\hline \multirow{5}{*}{$\begin{array}{l}\text { Geograpsus lividus } \\
\text { "cangrejo" }\end{array}$} & Ara1-All & + & - & $+t$ & - & - & - \\
\hline & Ara2-All & ++ & $\cdot$ & ++ & . & - & . \\
\hline & Ara3-All & +++ & - & ++ & - & - & $\cdot$ \\
\hline & Ara4-All & + & - & + & $\cdot$ & - & - \\
\hline & Ara5-All & $\cdot$ & +++ & $\cdot$ & $+t$ & ++ & - \\
\hline \multirow{2}{*}{$\begin{array}{l}\text { Aulacomya ater } \\
\text { "choro" }\end{array}$} & Cho1-All & + & ++ & ++ & + & + & - \\
\hline & Cho2-All & ++ & $\cdot$ & · & - & - & - \\
\hline
\end{tabular}

1: AMILASA; 2: CASEINASA; 3: TWEEN-ESTERASA; 4: DNASA; 5: GELATINASA; 6: AGARASA

(") Halos de actividad enzimática (en $\mathrm{mm}$ de diámelro): $\quad+=<8 \mathrm{~mm} \quad++=8.16 \mathrm{~mm} \quad+++=>16 \mathrm{~mm}$ (i) alga (muestra referencial). 
Tabla 3. Actividad multienzimática de 10 bacterias marinas con identificación preliminar y aisladas de Argopecten purpuratus "concha de abanico" y Crassostrea gigas "ostra" en cultivos

\begin{tabular}{|c|c|c|c|c|c|c|c|}
\hline CEPAS & Gelatina & Caseína & Almidón & DNA & Tween-80 & Lecitina & GENERO \\
\hline CA. $5^{*}$ & ++ & +++ & ++ & ++ & ++ & + & $\begin{array}{c}\text { No } \\
\text { identificado }\end{array}$ \\
\hline CA. 6 & - & ++ & $+t+$ & +++ & + & + & Vibrio \\
\hline CA.8 & + & + & ++ & + & - & - & Flexibacter \\
\hline CA.21 & ++ & ++ & + & t+ & ++ & ++ & Vibrio \\
\hline CA. 25 & ++ & +++ & ++ & - & +++ & ++ & Moraxella \\
\hline CA.34 & - & ++ & + & ++ & ++ & + & Vibrio \\
\hline OS.13** & +++ & +++ & ++ & ++ & ++ & ++ & Vibrio \\
\hline OS.16 & ++ & $++t$ & + & ++ & +++ & ++ & $\begin{array}{c}\text { Flavobact- } \\
\text { erium }\end{array}$ \\
\hline OS.26 & ++ & +++ & ++ & + & ++ & ++ & $\begin{array}{c}\text { Flavobact- } \\
\text { erium }\end{array}$ \\
\hline OS.49 & ++ & + & - & - & ++ & + & Vibrio \\
\hline
\end{tabular}

* Cepas CA proceden de Argopecten purpuratus "concha de abanico"

* Cepas OS proceden de Crassostrea gigas "ostra".

- $\quad: \sin$ actividad

$+\quad:<8 \mathrm{~mm}$

$+\quad: 8-16 \mathrm{~mm}$

$\mathrm{H+}:>16 \mathrm{~mm}$

Tabla 4. Frecuencia de bacterias marinas con actividad exoenzimática expresada en porcentaje

\begin{tabular}{llll}
\hline \multirow{2}{*}{ SUBSTRATO } & \multicolumn{1}{c}{ EEC } & \multicolumn{2}{c}{$\begin{array}{c}\text { Cepas con actividad } \\
\text { enzimática }\end{array}$} \\
\cline { 3 - 4 } & & $64 / 102$ & 62,74 \\
\hline CASEÍNA & Caseinasa & $59 / 102$ & 57,84 \\
TWEEN-80 & Tween-esteresa & $54 / 102$ & 52,94 \\
ALMIDÓN & Amilasa & $39 / 102$ & 38,23 \\
GELATINA & Gelatinasa & $34 / 102$ & 33,33 \\
DNA & DNAsa & $5 / 92$ & 5,43 \\
AGAR & Agarasa & $9 / 10$ & 90,0 \\
LECITINA & Lecilinasa & & \\
\hline
\end{tabular}




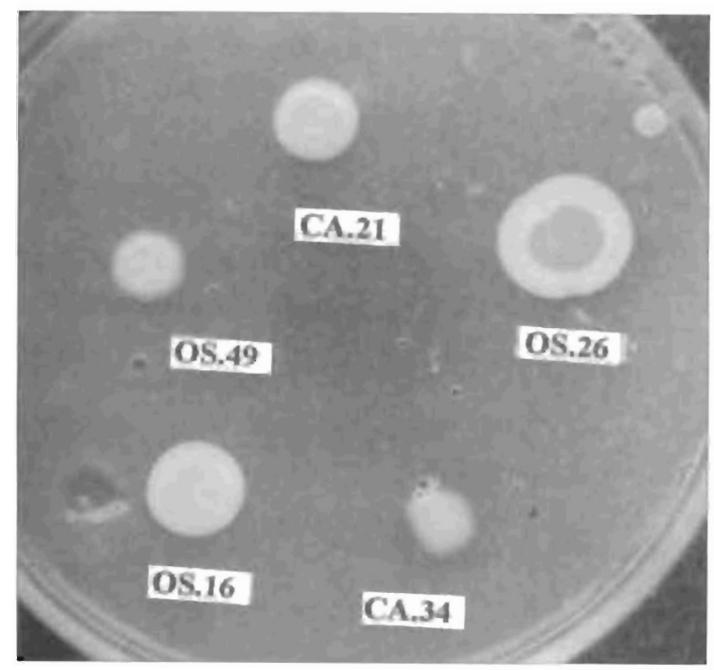

Fig. 1. Producción de lecitinasa y fosfolipasa por las cepas CA.21, OS.26, CA.34, OS.16 y OS.49. La actividad de la primera se evidencia por la formación de un halo blanquecino (muy notorio en la cepa OS.26) y y la segunda por un halo transparente alrededor de las colonias.

Fig. 2. Producción tween esterasa por las cepas CA.21, OS.26, CA.34, OS.16 y OS.49. La formacion de un halo opaco alrededor de las colonias evidencia la actividad de esta enzima.
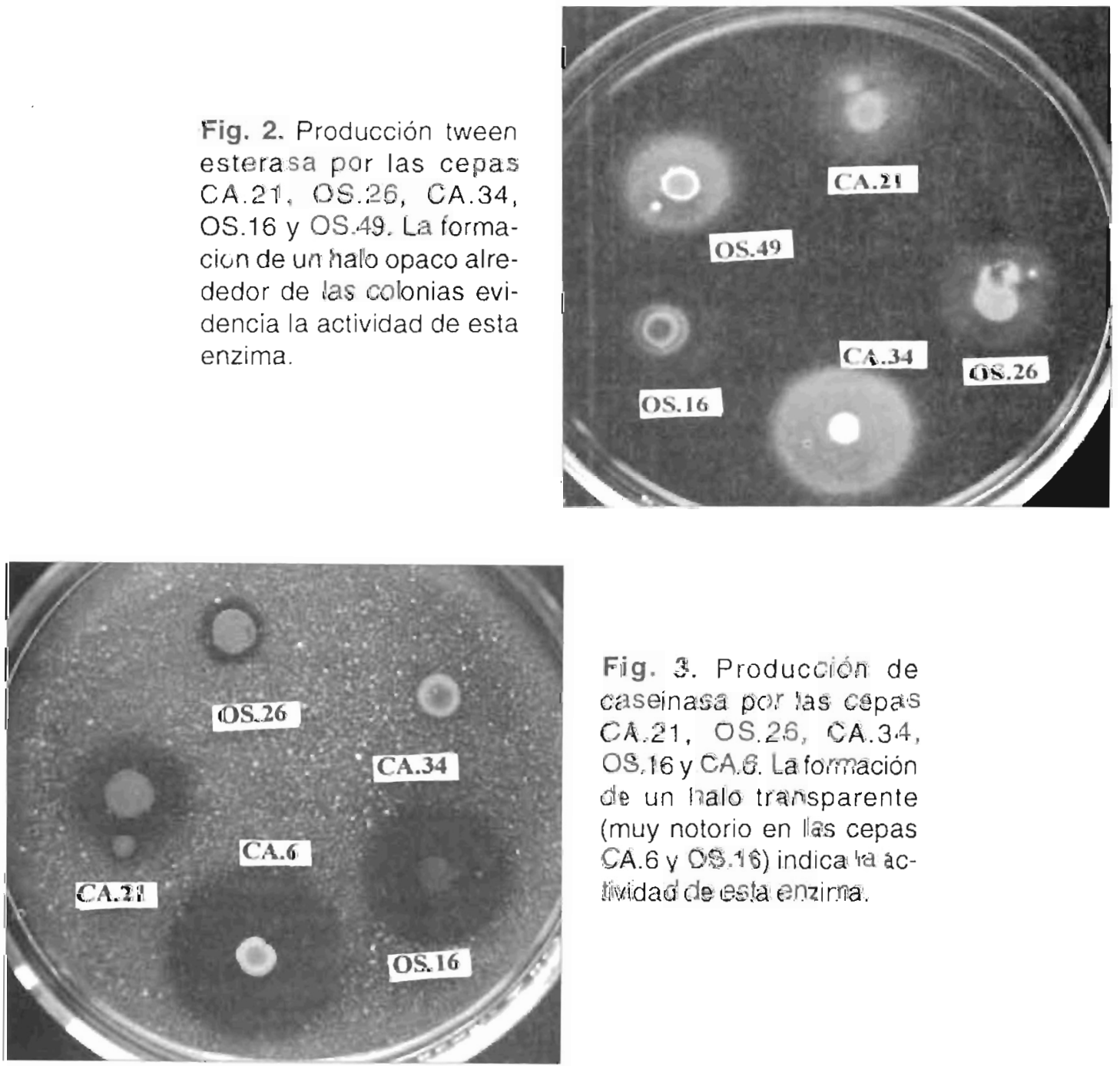

Fig. 3. Producción de caseinasa por las cepas CA.21, OS.26, CA.34, OS.16 y CA.6. La formación de un halo transparente (muy notorio en las cepas CA.6 y OS.16) indica la actividad de esta enzima. 


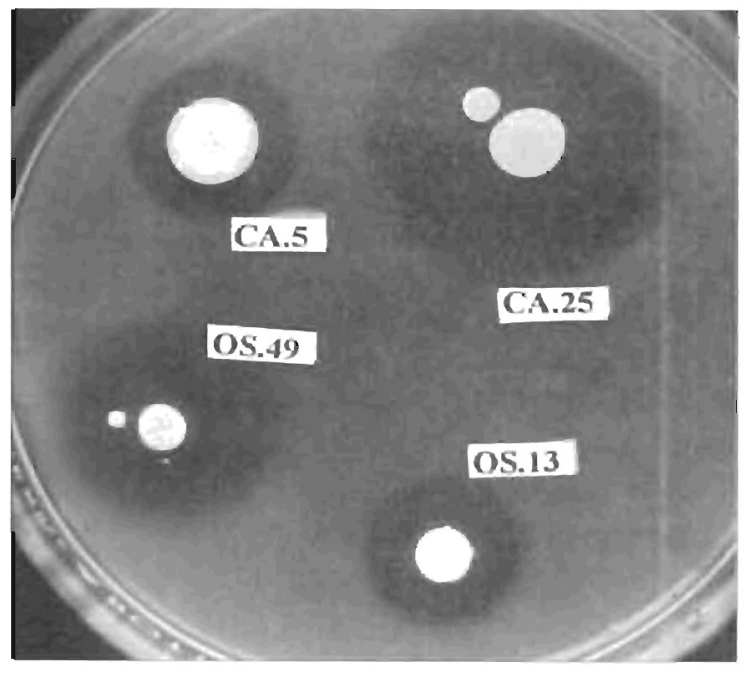

Fig. 4. Hidrólisis de DNA por las cepas CA.5, CA.25, OS.13 y OS.49. El halo transparente alrededor de las colonias indica actividad de la DNAsa, muy evidente en la cepa CA.25.

Fig. 5. Hidrólisis de la Gelatina por las cepas CA.21, OS.26, CA.34, OS.16 y CA.6, cuyas colonias presentan un halo transparente como producto de la actividad enzimática de la gelatinasa microbiana.
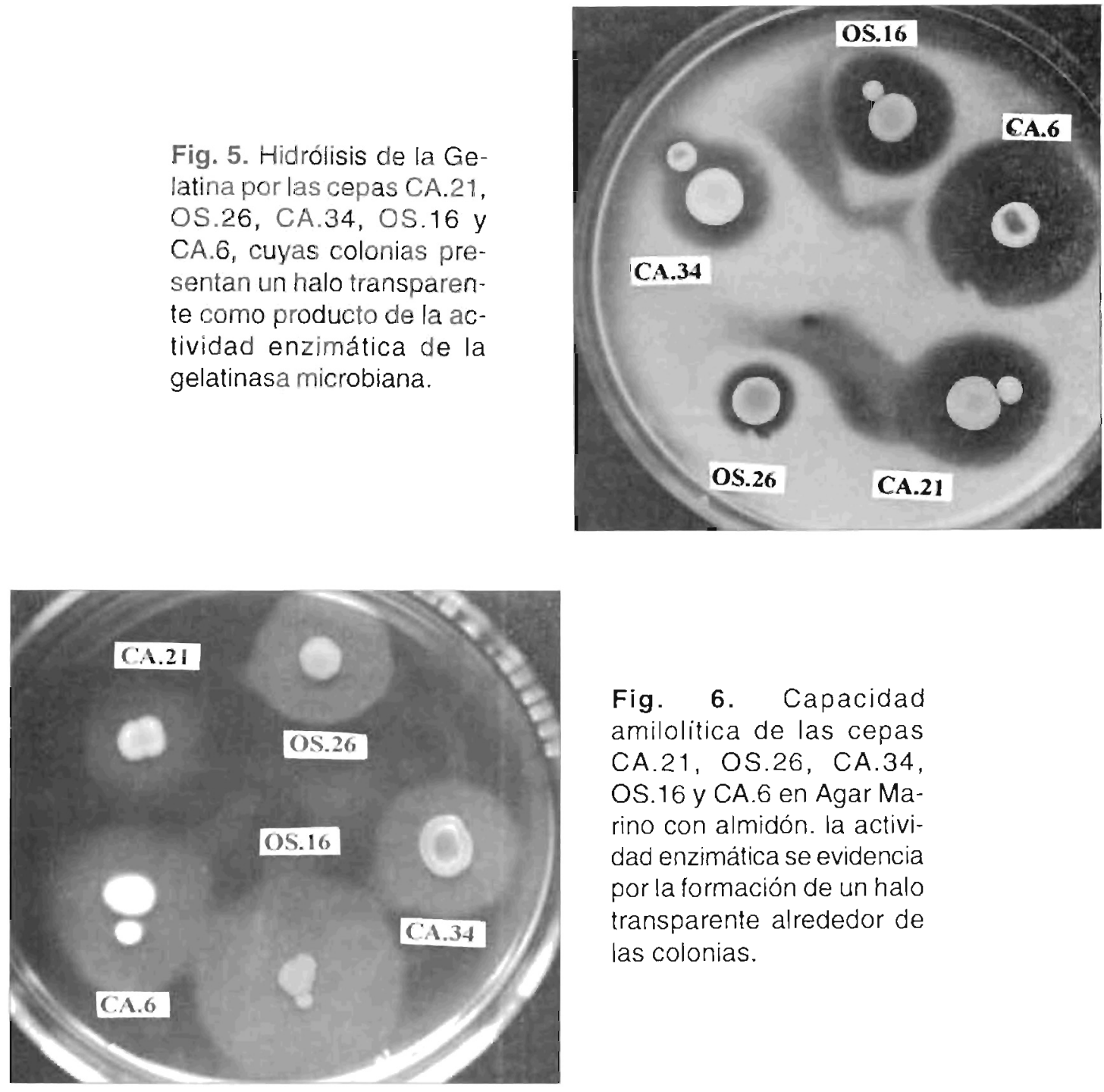

Fig. 6. Capacidad amilolitica de las cepas CA.21, OS.26, CA.34, OS.16 y CA.6 en Agar Marino con almidón. la actividad enzimática se evidencia por la formación de un halo transparente alrededor de las colonias. 